\title{
Numerical Design of Sound-Insulating Material with Combined Cavities of Mixed Sizes
}

\author{
Xu-wen Wang $\left(D,{ }^{1}\right.$ Shao-wei Li, ${ }^{2}$ Wei Cheng, ${ }^{1}$ Jing-rui Li, ${ }^{1}$ Zhi Fang, ${ }^{3}$ and Bing Wang ${ }^{1}$ \\ ${ }^{1}$ Luoyang Ship Material Research Institute, Luoyang 471039, China \\ ${ }^{2}$ Bohai Shipyard Group Co. Ltd, Huludao 125004, China \\ ${ }^{3}$ Huazhong University of Science and Technology, Wuhan 430074, China
}

Correspondence should be addressed to Xu-wen Wang; 31866413@qq.com

Received 12 December 2019; Accepted 18 February 2020; Published 22 June 2020

Academic Editor: Mario Terzo

Copyright ( $\odot 2020 \mathrm{Xu}$-wen Wang et al. This is an open access article distributed under the Creative Commons Attribution License, which permits unrestricted use, distribution, and reproduction in any medium, provided the original work is properly cited.

In view of the low-frequency acoustic insulation performance and poor pressure resistance of a single cavity at present, finite element software COMSOL is used to establish an analysis model to calculate the sound transmission loss (TL) and compression performance of the sound-insulating material with combined cone cavities. The effectiveness of the numerical method is validated by comparing with the experimental results, and influence of the structural parameters of the cone cavity, the perforation, and the mixed cavity shape on the sound transmission loss is investigated.

\section{Introduction}

The sound absorption and noise reduction of underwater submersible have been paid great attention by many countries. At present, acoustic covering technology is the only comprehensive technology that can reduce the target intensity of underwater submersible and suppress the radiation noise of underwater submersible. In the Second World War, the German navy for the first time added a synthetic rubber soundproof material named "Alberich" [1] to the shell of the submarine, which was the earliest resonant acoustic covering with a short cylindrical cavity.

After half a century of development, the resonator cavity has been spherical, conical, circular, and exponential [2-8]. At present, the underwater resonant acoustic overlay with the exponential cavity is the most widely used. Ivansson [9] compared anechoic coatings containing cylindrical holes having different cross-sectional shapes (circle, ellipse, and superellipse) and used the Markov-chain Monte Carlo method to obtain optimal solutions. Shang et al. [10] found that conical holes are superior to cylindrical holes for enhancing the sound absorption performance of anechoic layers.
Ye et al. [11] established a theoretical model to evaluate the sound absorption performance of underwater anechoic layers containing periodically distributed axial holes. Three different types of axial holes are considered: the cylindrical, the conical, and the horn-shaped one. Results obtained with full finite element simulations are used to validate the model predictions, and the effect of the hole shape at different incidence angles is studied. The results show that two new absorption peaks appear since the oblique incidence excites two horizontal modes. Among the three hole types, the horn one achieves the best absorption performance at relatively low frequencies both in normal incidence and in oblique incidence.

To explore better decoupling performance, an optimal design of acoustic coating with complex-shaped cavities is attempted by Huang et al. [12]. An analytical vibroacoustic model is developed for the prediction of the sound radiation from an infinite plate covered with an equivalent fluid layer (as a replacement of original coating) and immersed in water. Numerical examples are provided to verify the equivalent fluid model. Based on combining use of the analytical vibroacoustic model and a differential evolution algorithm, optimal designs for acoustic coatings with cavities 
are conducted. A number of connected cavity structures are arranged along the thickness of the acoustic overlay so that multiple resonances of sound waves occur in multiple cavities, increasing the acoustic insulation performance of the acoustic overlay. Numerical results demonstrate that the decoupling performance of acoustic coating can be significantly improved by employing special axisymmetrical cavities as compared to traditional cylindrical cavities.

A paper published in Applied Acoustics [13] proposed a new kind of cavity structural-acoustic overlay. It was named an inverted cyathiform cavity, that is, an inverted conical structural cavity. Compared with the coating with isochoric cylindrical cavities, the optimized coating can achieve better sound absorption in low frequencies. This phenomenon is attributed to the larger pore-aperture radius of the optimized cavity than that of the cylindrical cavity. Besides, the frequency-dependent parameters of the rubber material play an important role in the broadband high absorption of the optimized coating.

In the above literature studies, many acoustic structures of acoustic overlay have been tried, but there is still a large space for optimization, and the influence of structural parameters on its sound absorption performance needs to be further analyzed and refined.

In this paper, commercially available FE code COMSOL Multiphysics is used to obtain the sound transmission loss and deformation of the sound-insulating material under a hydrostatic pressure, and a combined cavity structure is proposed. The effectiveness of the analysis method is validated by comparing with the experimental results, and the influence of the structural parameters of the cone cavity, the perforation, and the mixed cavity shape on the sound transmission loss is investigated.

\section{Numerical Calculation Model}

The finite element simulations are carried out by using COMSOL. Due to periodicity, a single cell is simulated using "free triangular" elements. In the "physics" section, "acoustic-elastic wave interaction" is adopted. The rubber domain is simulated by the "elastic wave" component, while the air domain in the hole as well as the external water domain are simulated by the "pressure acoustic" component.

In COMSOL, the acoustic sound tube is simulated to calculate the sound transmission loss of the acoustic cover material. In software COMSOL, the PML (perfectly matched layer) is used to model an open or infinite domain for both the elastic waves and the pressure waves, which can simulate the nonreflective boundary conditions. As the PML boundary condition is used in the simulation, the end termination is designed to unreflected sound wave energy. So, the sound pressure of the sound filed in the downstream of the setup can be explained by one hydrophone, namely, the three-hydrophone technique. Comparing with the fourhydrophone method, the model using the three hydrophones can be less time consuming. The three-hydrophone transfer function method is used in the simulation. The calculation principle of the three-hydrophone transfer function is shown in Figure 1. The entrance sound source generates the signal. The incident wave $p_{i}$ is generated by the sound source at the entrance, propagating in the incident tube. Two sound waves are formed due to the absorption of the test sample when the forward-propagating incident wave $p_{i}$ encounters the test sample, and one is noted by the transmitted wave $p_{t}$, which continues to propagate forward in the transmission tube. The other is marked by the reflected wave $p_{r}$ due to the reflection of the test sample, which propagates in the opposite direction in the incident tube. After reaching the acoustic end with perfect absorption performance, the sound wave at the end is completely absorbed. In COMSOL software, the PML nonreflective terminal is used to perfectly match the layer boundary conditions to simulate a completely nonreflective end.

If the measured sound pressure at the hydrophone $P_{1}$ is noted as $p_{1}$, the measured sound pressure at the hydrophone $P_{2}$ is noted as $p_{2}$, and the measured sound pressure at the hydrophone $P_{3}$ is noted as $p_{3}$, the relationship between the wave sound pressure $p_{i}$, the transmitted wave sound pressure $p_{t}$, and the reflected wave sound pressure $p_{r}$ with the measured sound pressures $p_{1}, p_{2}$, and $p_{3}$ can be obtained from the acoustic wave equation:

$$
\left\{\begin{array}{l}
P_{1}=P_{i} e^{j k\left(S_{1}+L_{1}\right)}+P_{r} e^{-j k\left(S_{1}+L_{1}\right)}, \\
P_{2}=P_{i} e^{j k L_{1}}+P_{r} e^{-j k L_{1}}, \\
P_{3}=P_{t} e^{j k\left(L_{2}+d\right)} .
\end{array}\right.
$$

The simplified solutions of the three formulas above are as follows:

$$
\begin{aligned}
P_{i} & =\frac{P_{1}-P_{2} e^{-j k S_{1}}}{e^{j k S_{1}}-e^{-j k S_{1}}} e^{-j k L_{1}}, \\
P_{t} & =e^{-j k\left(L_{2}+d\right)}
\end{aligned}
$$

The sound pressure transmission coefficient $t_{p}$ is expressed by the incident wave sound pressure and transmission wave sound pressure as follows:

$$
t_{p}=\frac{P_{t}}{P_{i}} .
$$

The sound transmission loss of the test sample is obtained as follows:

$$
\mathrm{TL}=-20 \lg \left|t_{p}\right|
$$

In the present study, with focus placed on the representative underwater applications, the thickness of the anechoic layer $l_{a}$ is fixed at $30 \mathrm{~mm}$ and the height of the hole $l_{h}$ at $23 \mathrm{~mm}$.

\section{Results and Discussion}

3.1. Numerical Calculation. To validate the numerical calculation model, the sound transmission loss of the underwater sound absorption coating presented in [14] is calculated and compared with the simulation results [14] and the experimental measurements provided by [15]. The 


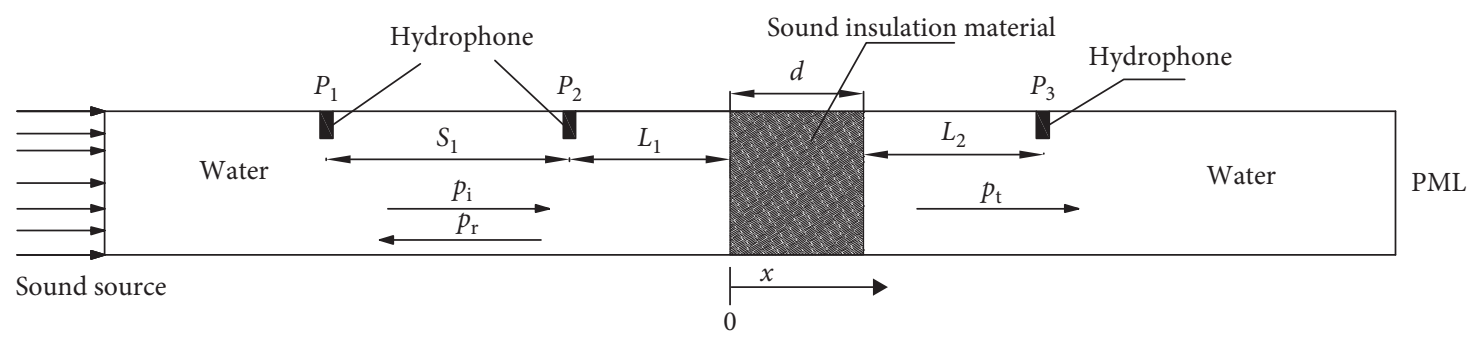

FIgURE 1: The three-hydrophone transfer function method is used in the simulation.

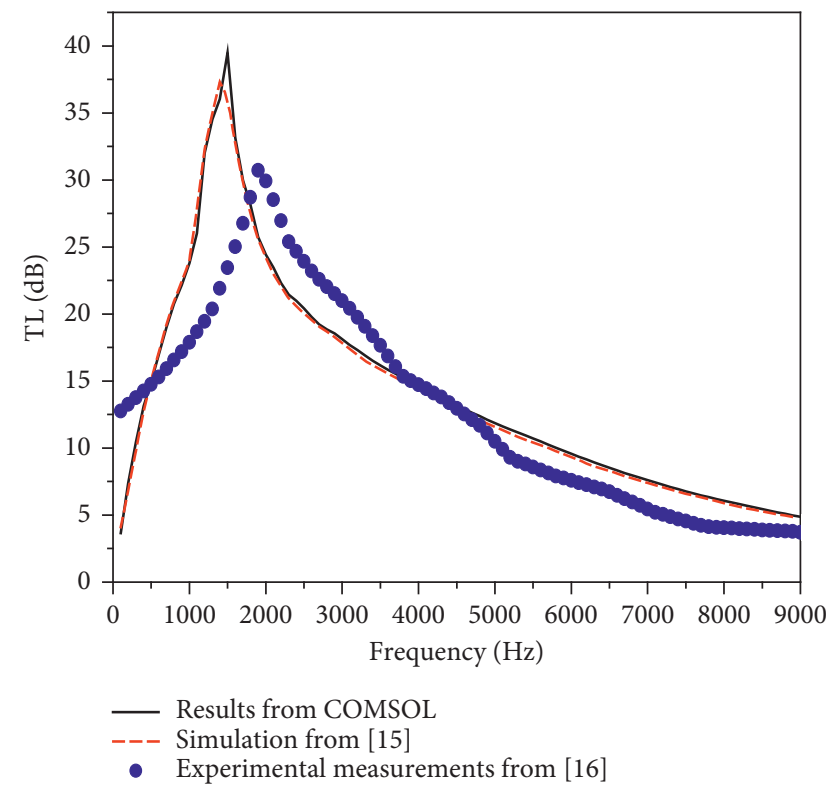

FIGURE 2: Validation of the simulation calculation from COMSOL.

four-hydrophone technique is used in the experiment, and the sound-absorption wedge is applied at the end of the tube to absorb the transmitted sound wave energy. The comparison of the sound transmission loss results is shown in Figure 2. The finite element simulation results of the COMSOL model agree well with the finite element simulation results in the literature, and the trend is the same as the experimental results, but there are some differences. The existence of this difference may be due to the inconsistency between the setting of the nonreflecting end in the simulation calculation and the setting during the measurement. It may also be due to the coupling effect between the underwater acoustic tube wall and the sound insulation material and the water medium in the experiment, which cannot be considered in the simulation calculation. The change of the cavity type does not affect the accuracy of the simulation calculation method. It can be verified that the sound transmission loss calculation obtained by COMSOL is credible.

3.2. Effects of the Structural Parameters on the Sound Transmission Loss. The combination of the cone cavity is designed into a combined cavity structure to calculate the sound transmission loss and deformation under pressure. A
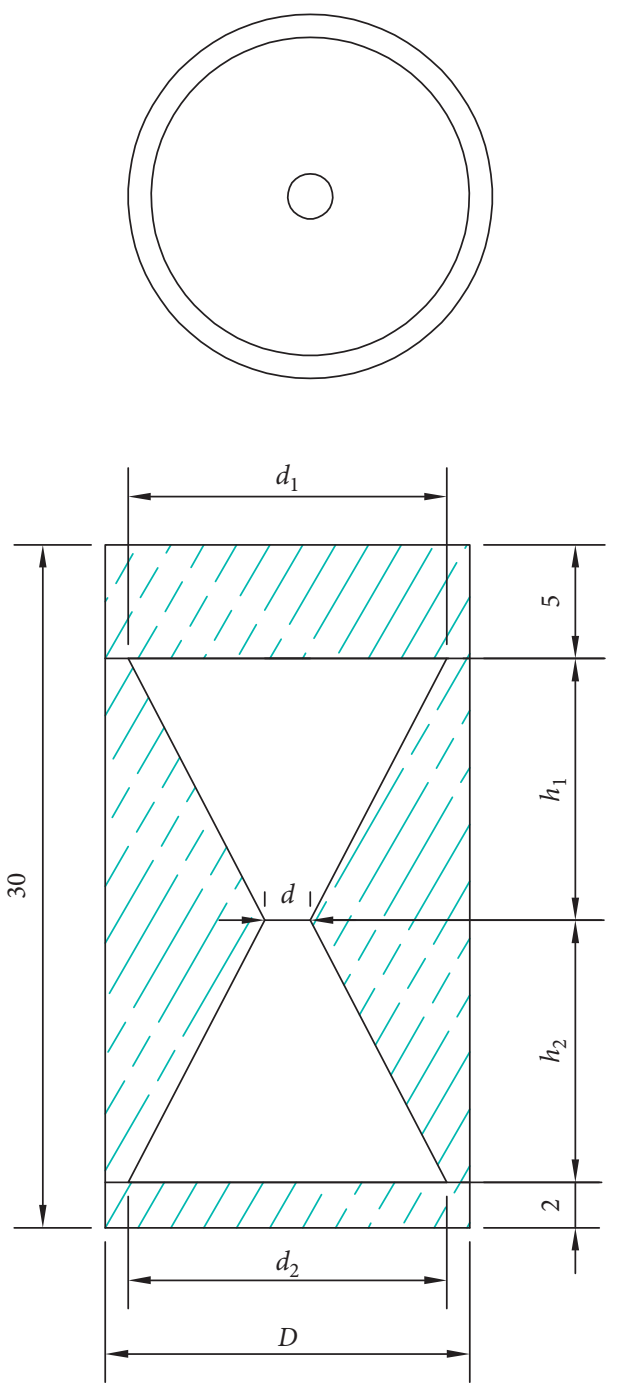

FIGURE 3: Schematic diagram of the single cell structure of the sound insulation material with the combined cavity.

schematic diagram of the combined cavity structure is shown in Figure 3.

The influence of structural parameters on the sound transmission loss is analyzed by changing the diameter of the sound-insulating material cell and the maximum diameter of the cavity. In the analysis of this section, the diameter of the small section of the cone is taken as $d=2 \mathrm{~mm}$, the height of the upper and lower cones is both taken as $h_{1}=h_{2}=11.5 \mathrm{~mm}$, the radius of the larger section of the cone 


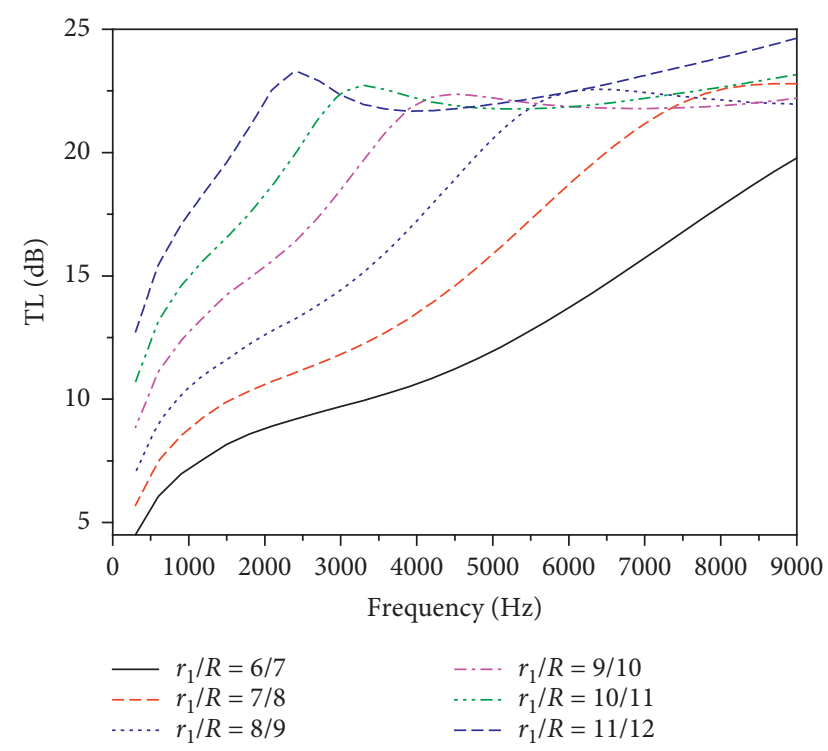

Figure 4: Effect of the diameter of the sound-insulating material cell and the maximum diameter of the cavity on TL.

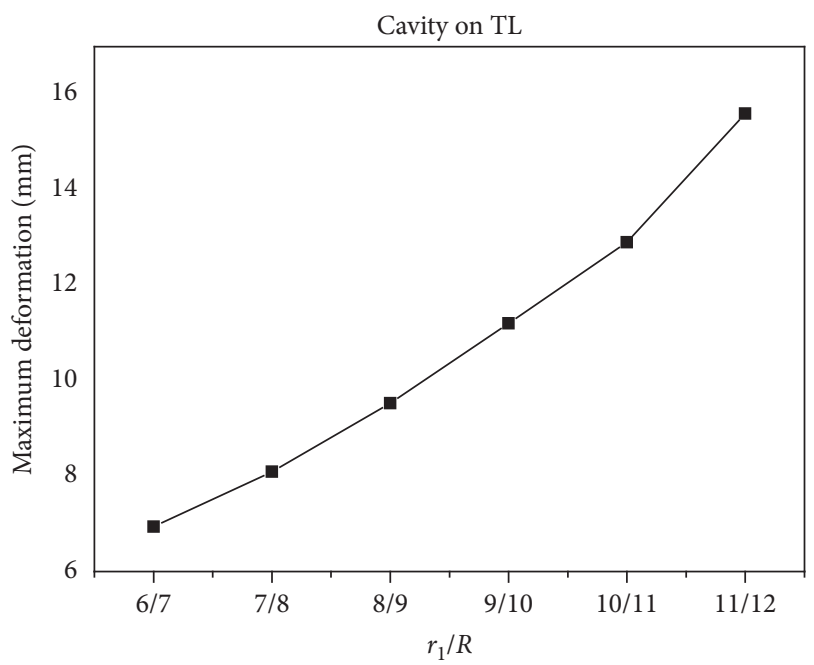

FIGURE 5: Effect of the diameter of the sound-insulating material cell and the maximum diameter of the cavity on deformation under the hydrostatic pressure of $3 \mathrm{MPa}$.

$r_{1}=0.5 d_{1}=0.5 d_{2}$ is taken as $6 \mathrm{~mm}, 7 \mathrm{~mm}, 8 \mathrm{~mm}, 9 \mathrm{~mm}$, $10 \mathrm{~mm}$, and $11 \mathrm{~mm}$ separately, and correspondingly, the radius of the single cell of the sound insulation material is taken as $7 \mathrm{~mm}, 8 \mathrm{~mm}, 9 \mathrm{~mm}, 10 \mathrm{~mm}, 11 \mathrm{~mm}$, and $12 \mathrm{~mm}$, respectively. The sound transmission loss and the maximum deformation under the hydrostatic pressure of $3 \mathrm{MPa}$ results are calculated with different ratios of the maximum diameter of the cavity to the diameter of the sound insulation material cell unit, and the comparison curves are shown in Figures 4 and 5 , respectively.

It can be seen from Figures 4 and 5 that when the ratio of the maximum cross section of the cavity to the cross-sectional area of the unit cell is larger, the sound transmission loss of the sound-insulating material is better, the peak of the

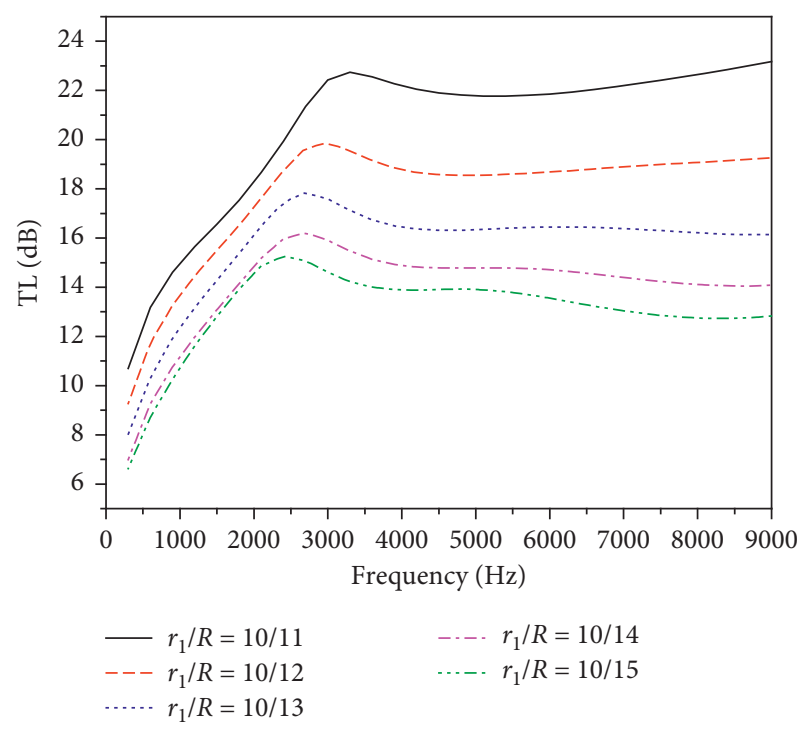

FIGURE 6: TL curves with different distances between two adjacent cavities.

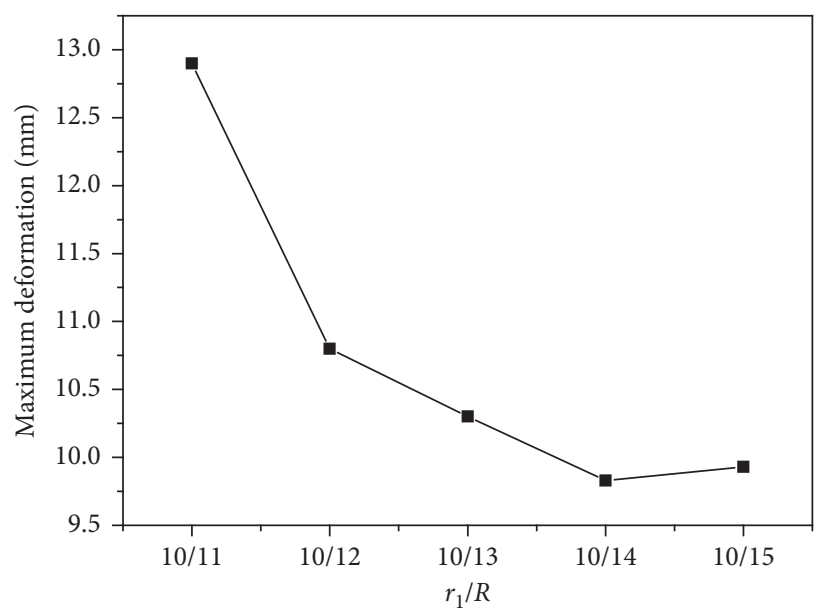

FIgURE 7: Deformation curves with different distances between two adjacent cavities.

sound transmission loss is gradually appeared, and the peak frequency moves to the lower frequency. However, the larger the ratio of the cross-sectional area, that is, the larger the cavity perforation rate, the worse the pressure resistance of the sound-insulating material and the bigger the deformation under hydrostatic pressure. When the maximum crosssectional radius of the cavity structure is $10 \mathrm{~mm}$ and the unit cell is $11 \mathrm{~mm}$, the sound insulation curve peaks around $3,000 \mathrm{~Hz}$.

Reducing the perforation rate of the sound-insulating material can improve the withstand voltage performance, but the sound-insulating performance is affected. On the basis of the fixed cavity volume, the maximum cross-sectional diameter of the cavity is fixed as $10 \mathrm{~mm}$, and the change trend of sound transmission loss performance and compression performance of the sound-insulating material is investigated with increased unit cell volume. The changes 

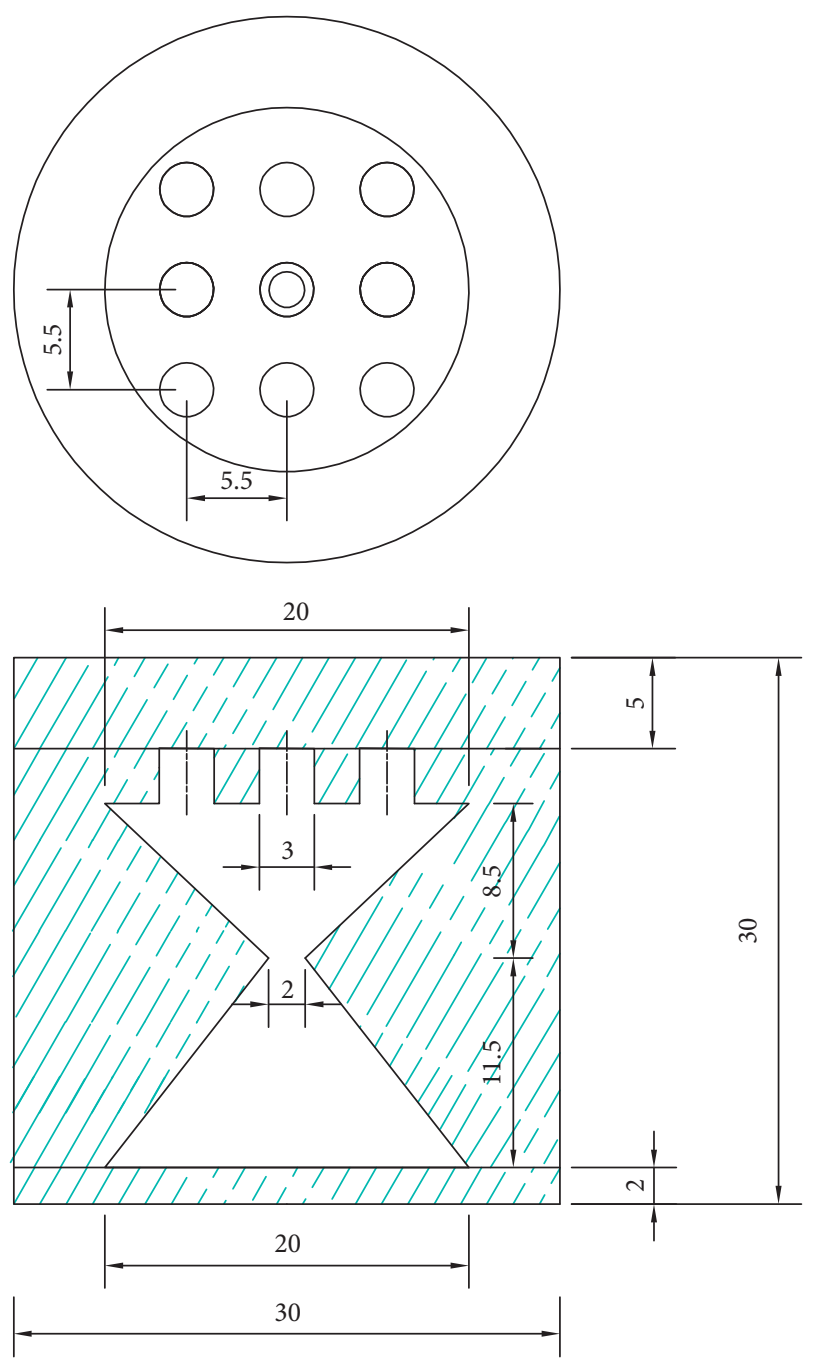

FIGURE 8: Structure of the sound-insulating material with the perforated combined cavity.

in the sound transmission loss curves and the deformation amount are shown in Figures 6 and 7, respectively.

It can be seen from Figures 6 and 7 that when the cavity volume is fixed, the peak value in the sound transmission loss curve shifts to the lower frequency with increased cell unit volume, and the overall sound insulation performance becomes worse. However, as the unit cell volume increases, the amount of deformation of the sound-insulating material under a hydrostatic pressure of $3 \mathrm{MPa}$ becomes smaller. Therefore, the design of the cavity should balance the sound insulation performance and the compression performance, and the volume of the cavity and the distance between two adjacent cavities should be rationally designed according to the frequency range to be improved.

3.3. Effects of Perforation on the Sound Transmission Loss. It can be known from Section 3.2 that reducing the volume of the cavity can improve the compression performance of the sound-insulating material. In order to ensure the sound transmission loss of the sound insulation material at the low

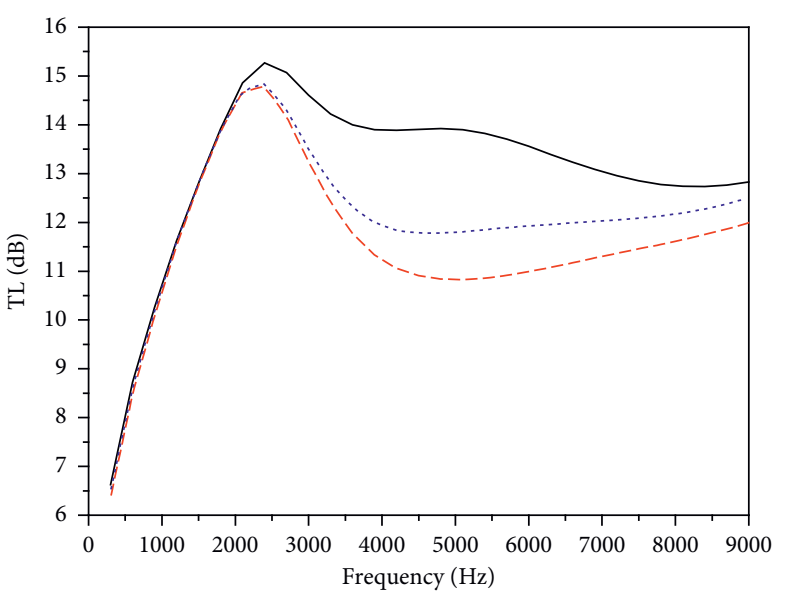

\footnotetext{
Combined cavity: $r_{1} / R=10 / 15, h_{1}=h_{2}=11.5 \mathrm{~mm}$, maximum deformation: $9.93 \mathrm{~mm}$

- - Combined cavity: $r_{1} / R=10 / 15, h_{1}=8.5 \mathrm{~mm}, h_{2}=11.5 \mathrm{~mm}$, maximum deformation: $6.22 \mathrm{~mm}$

-... Combined cavity with perforation: $r_{1} / R=10 / 15, h_{1}=8.5 \mathrm{~mm}$, $h_{2}=11.5 \mathrm{~mm}$; maximum deformation: $7.15 \mathrm{~mm}$
}

FIgURE 9: Comparison of the sound transmission loss curve between the combined cavity and the perforated combined cavity.

frequency, the combined cavity structure is changed on the basis of Figure 3. The maximum cross-sectional area of the cone cavity is not changed to ensure that the resonance peak frequency is fixed, and the volume of the combined cavity is reduced by shortening the height of the conical cavity. It is known that the smaller cavity volume can reduce the amount of sound insulation of the sound-insulating material as a whole, so it is selected to perforate the side of the sealing layer to increase the high-frequency sound insulation. The structure of the designed perforated combined cavity is shown in Figure 8. It can be seen from the comparison of the sound transmission loss curve shown in Figure 9 that the peak frequency in the sound transmission loss curve does not change when the volume of the cone cavity is reduced with the fixed maximum cross-sectional area of the cavity. The perforation is punched in the rubber material near the sealing layer, and compared with nonperforation, the peak frequency is not changed, and the sound transmission loss performance in the medium- and high-frequency range can be improved.

\subsection{Effects of the Mixed Cavity Shape on the Sound Trans-} mission Loss. On the basis of the combined cavity shape, cylindrical or conical cavities are added to the side to form a mixed cavity shape, and the sound insulation performance and compression performance are investigated. Figure 10 shows the structural schematic view and related dimensions of the perforated hybrid cavity-shaped sound-insulating materials. The calculated sound transmission loss curves are displayed in Figure 11.

In the study of this section, the fixed radius of the unit cell of the sound insulation material is $R=15 \mathrm{~mm}$, the maximum radius of the upper and the lower cone cavity is $r=10 \mathrm{~mm}$, the minimum cross-sectional diameter of the 

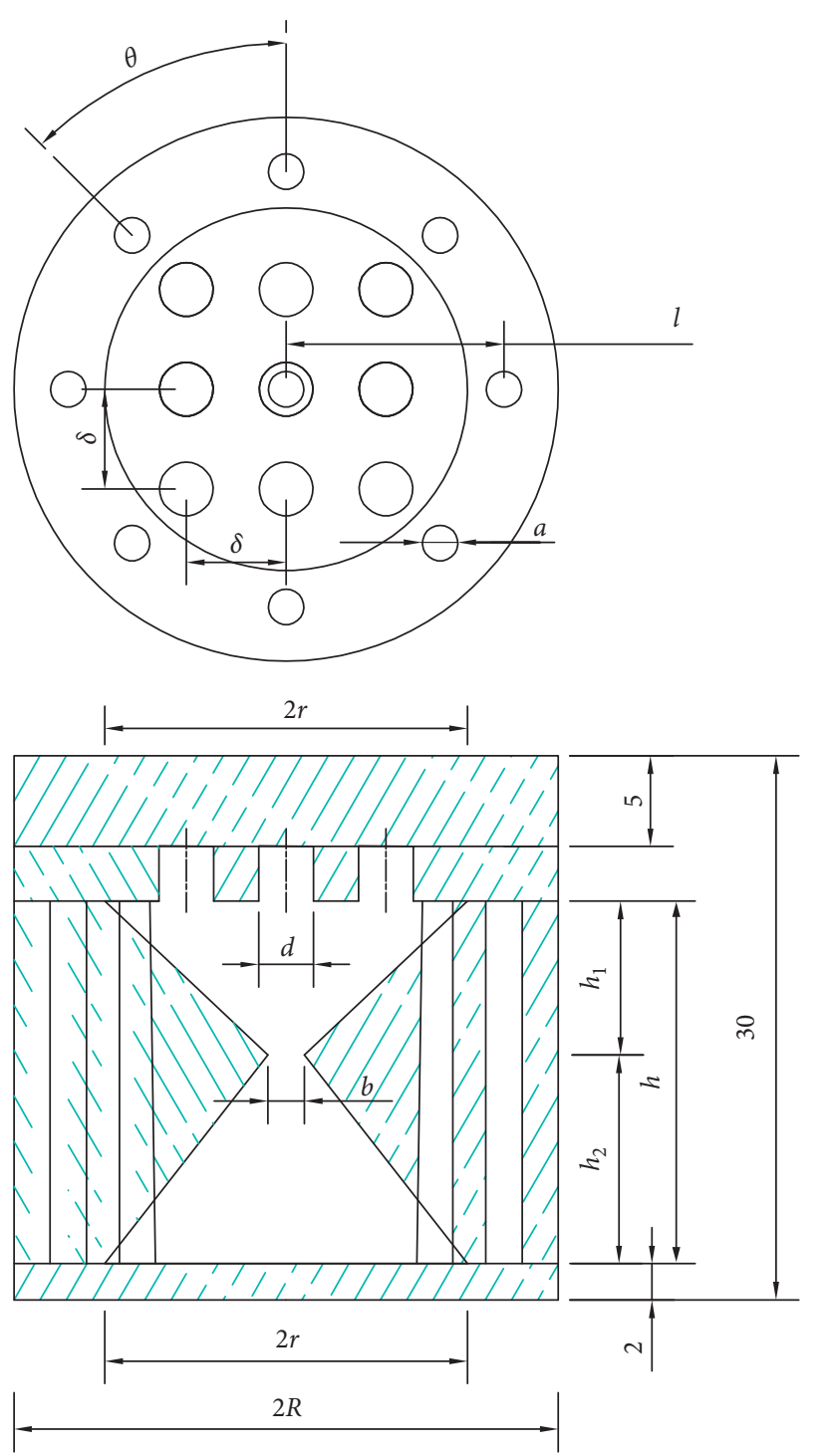

FIgURE 10: Structure of the sound-insulating material with the perforated mixed cavity.

cone cavity is $b=2 \mathrm{~mm}$, and the heights of the upper and the lower cone cavity are $h_{1}=8.5 \mathrm{~mm}$ and $h_{2}=11.5 \mathrm{~mm}$, respectively. The perforation aperture above the upper cone cavity is $d=2 \mathrm{~mm}$, and the holes are arranged in a square with a spacing of $\delta=5.5 \mathrm{~mm}$.

$\mathrm{t}$ can be seen from Figure 11 that the addition of axial cylindrical cavities around the combined cone cavity increases the effective cross-sectional area of the cavity, which can improve the sound transmission loss in the low-frequency range and cause the peak to move toward the lower frequency. Since the perforation layer is relatively thin, the influence of the perforation aperture and the number of perforations on the perforation volume is small, and thus, the effect on the sound insulation performance is not obvious. Therefore, based on the parameters of the fixed perforation layer, the diameter size and height of the side cylindrical cavity are changed, and the influence law on the sound insulation performance is analyzed.

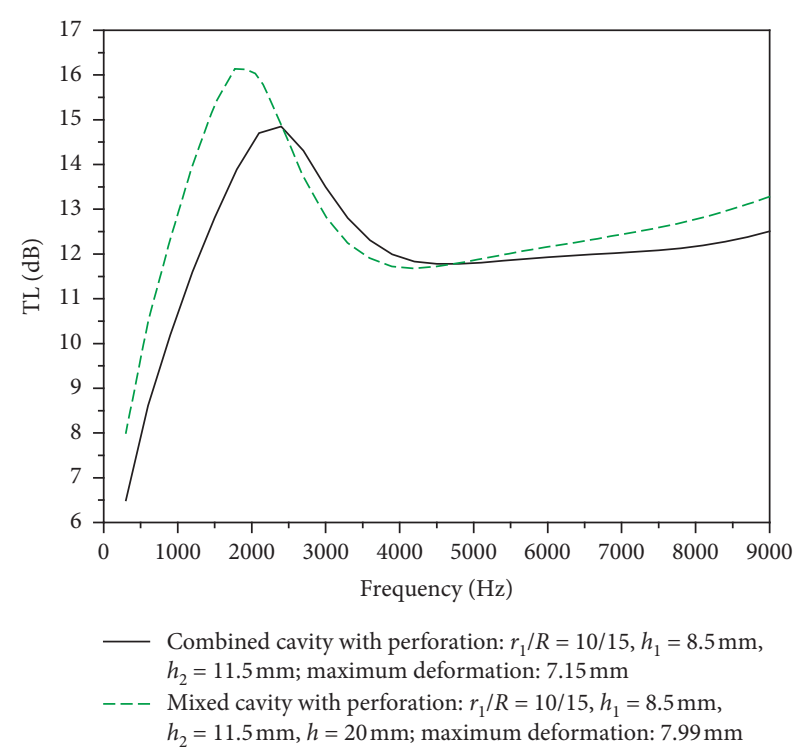

FIgURE 11: Comparison of the sound transmission loss curve between the combined cavity and the mixed cavity.

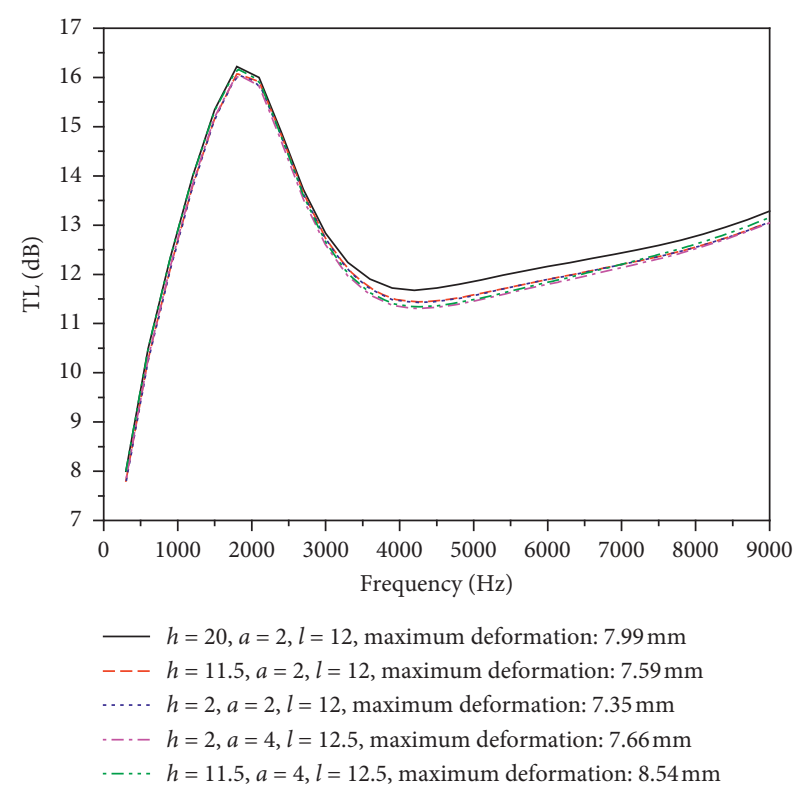

FIGURE 12: Effect of the height and aperture of side-branch mixed cavities on the TL and deformation.

Figure 12 shows the sound transmission loss and the maximum deformation of the sound-insulating material under a hydrostatic pressure of $3 \mathrm{MPa}$ with different heights and apertures of side-branch mixed cavities when the number of cavities is fixed to 8 . It can be seen from the graph that changing the height and aperture of the side-branch cavity has little influence on sound insulation volume and basically no influence on the sound insulation performance before the peak frequency. When the volume of the sidebranch cavity increases, the sound insulation performance will be slightly improved in the medium-high frequency, but the pressure tolerance performance will not be ignored. 
Therefore, the design of the side cavity needs to balance the sound insulation performance and the pressure resistance performance and comprehensively consider the difficulty of processing.

\section{Conclusions}

In view of the low-frequency acoustic insulation performance and poor pressure resistance of a single cavity at present, finite element software COMSOL is used to establish an analysis model to calculate the sound transmission loss and compression performance of the soundinsulating material with combined cone cavities. The effectiveness of the analysis method is validated by comparing with the experimental results, and influence of the structural parameters of the cone cavity, the perforation, and the mixed cavity shape on the sound transmission loss is investigated.

The results show that (1) when the volume of the single cell unit of the sound insulation material is fixed, the larger the effective maximum cross-sectional area of the cavity part is, the better the sound transmission loss performance in the low-frequency range is; (2) when the effective volume of the cavity is fixed, as the volume of the sound insulation material increases, that is, the perforation rate decreases, the peak frequency in the sound transmission loss curve moves to the low frequency, and the overall sound transmission loss will be reduced; (3) with the fixed volume of the single cell unit of the sound insulation material and the effective maximum cross-sectional area of the cavity, drilling holes in the rubber material can improve the sound insulation performance in medium and high frequencies but has no effect on the sound transmission loss performance in the frequency band before the peak frequency; (4) with the fixed volume of the single cell unit of the sound insulation material and the effective maximum cross-sectional area of the cavity, a certain number of holes with small aperture around the cavity will improve the sound insulation performance at the low frequency and make the peak frequency move to the low frequency; (5) when the volume of the sound insulation material is fixed, the smaller the volume of all cavities, the better the pressure-resistant performance of the sound insulation material; and (6) the variation in the height and diameter of the side-branch holes has little influence on the sound insulation performance; however, as the height of the mixing cavity decreases, the pressure-resistant performance of the sound insulation material can be improved.

\section{Data Availability}

The data used to support the findings of this study are available from the corresponding author upon request.

\section{Conflicts of Interest}

The authors declare that they have no conflicts of interest.

\section{References}

[1] H. Oberst, Resonant Sound Absorbers, Elsevier, Amsterdam, Netherlands, 1957.

[2] E. Meyer, K. Brendel, and K. Tamm, "Pulsation oscillations of cavities in rubber," The Journal of the Acoustical Society of America, vol. 30, no. 12, pp. 1116-1124, 1958.

[3] G. Gaunaurd, "One-dimensional model for acoustic absorption in a viscoelastic medium containing short cylindrical cavities," The Journal of the Acoustical Society of America, vol. 62, no. 2, pp. 298-307, 1977.

[4] S. M. Ivansson, "Numerical modeling for design of viscoelastic coatings withfavorable sound absorbing properties," Nonlinear Analysis, vol. 63, pp. 1541-1550, 2005.

[5] S. M. Ivansson, "Sound absorption by viscoelastic coatings with periodically distributed cavities," The Journal of the Acoustical Society of America, vol. 119, no. 6, pp. 3558-3567, 2006.

[6] G. S. Sharma, A. Skvortsov, I. MacGillivray, and N. Kessissoglou, "Acoustic performance of gratings of cylindrical voids in a soft elastic medium with a steel backing," The Journal of the Acoustical Society of America, vol. 141, no. 6, pp. 4694-4704, 2017.

[7] W. L. Tang, S. P. He, and J. Fan, “Two-dimensional model for acoustic absorption of viscoelastic coating containing cylindrical holes," Acta Acustica, vol. 30, no. 4, p. 289-295, 2005.

[8] S. M. Ivansson, "Numerical design of alberich anechoic coatings with superellipsoidal cavities of mixed sizes," The Journal of the Acoustical Society of America, vol. 124, no. 4, pp. 1974-1984, 2008.

[9] S. M. Ivansson, "Markov-chain monte carlo identification of favorable design choices with application to anechoic coatings," The Journal of the Acoustical Society of America, vol. 135, no. 6, pp. 3338-3351, 2014.

[10] C. Shang, J. Z. Zhang, and Y. J. Wei, "Absorption characteristics of anechoic coating embedding frustum-of-a-cone cavities," Journal of Offshore Mechanics and Arctic Engineering, vol. 14, no. 12, pp. 1425-1431, 2010.

[11] C. Ye, X. Liu, F. Xin, and T. J. Lu, "Influence of hole shape on sound absorption of underwater anechoic layers," Journal of Sound and Vibration, vol. 426, pp. 54-74, 2018.

[12] M.-W. Huang, W.-J. Wu, X.-M. Lv, G.-Y. Yu, and J.-G. Zhang, "The role of 125 I interstitial brachytherapy for inoperable parotid gland carcinoma," Brachytherapy, vol. 17, no. 1, pp. 244-249, 2018.

[13] J. Zhong, H. Zhao, H. Yang, J. Yin, and J. Wen, "On the accuracy and optimization application of an axisymmetric simplified model for underwater sound absorption of anechoic coatings," Applied Acoustics, vol. 145, pp. 104-111, 2019.

[14] M. Tao, "Simplified model for predicting acoustic performance of an underwater sound absorption coating," Journal of Vibration and Control, vol. 20, no. 3, pp. 339-354, 2014.

[15] A. C. HladkyHennion and J. N. Decarpigny, "Analysis of the scattering of a plane acoustic wave by a doubly periodic structure using the finite element method: application to alberich anechoic coatings," Journal of the Acoustical Society of America, vol. 90, no. 6, pp. 3356-3367, 1991. 